\title{
Modeling Car-Following Behaviour of Turning Movements at Intersections with Consideration of Turning Radius
}

\author{
Fulu Wei, ${ }^{1,2}$ Yongqing Guo ${ }^{10},{ }^{1}$ Pan Liu, ${ }^{2}$ Zhenggan Cai, ${ }^{1}$ Qingyin Li, ${ }^{1}$ and Long Chen ${ }^{1}$ \\ ${ }^{1}$ Department of Transportation Engineering, Shandong University of Technology, Zibo 255000, China \\ ${ }^{2}$ Department of Transportation Planning and Management, Southeast University, Nanjing 210096, China \\ Correspondence should be addressed to Yongqing Guo; yongqing.guo@sdut.edu.cn
}

Received 8 June 2020; Revised 21 July 2020; Accepted 7 August 2020; Published 28 August 2020

Academic Editor: Ronghui Zhang

Copyright (c) 2020 Fulu Wei et al. This is an open access article distributed under the Creative Commons Attribution License, which permits unrestricted use, distribution, and reproduction in any medium, provided the original work is properly cited.

In order to deeply analyze and describe the characteristics of car-following behaviour of turning vehicles at intersections, the features and application conditions of classic car-following models were analyzed firstly. And then, through analysing the relationship between the maximum velocity of car-following vehicles and the turning radius of intersection, the differences in key variables between turning and straight car-following behaviour were identified. On the basis of Optimal Velocity (OV) model, a Turning Optimal Velocity (TOV) car-following model with consideration of turning radius and sideway force coefficient at intersections was developed. PreScan simulation was employed to build the scene of turning car-following process at an intersection. Based on linear stability analysis, the stability conditions of the TOV model were derived. And it was found that (1) the turning radius has a significant effect on the car-following behaviour of turning vehicles at intersections; (2) with the increase of the distance between vehicles, the driver's response sensitivity coefficient increases and then decreases and reaches the maximum value when the distance reaches the minimum safe distance; (3) with the increase of turning radius, the stability of the carfollowing fleet tends to decrease, and it is more likely to become a stop-and-go traffic flow. In addition, the numerical simulation results indicate that the TOV model can describe the car-following behaviour of turning vehicles more accurately with consideration of turning radius. The findings of this study can be used in the development of microscopic traffic simulation software and for improving traffic safety at intersections.

\section{Introduction}

With the rapid development of economy and the acceleration of urbanization, traffic pollution, traffic congestion, and other related problems have become social hotspots [1-4]. In order to solve these problems, traffic scholars and engineers have proposed a variety of traffic control strategies. In order to improve the handling performance of vehicle, a self-turning tyre friction control system was studied, and the hierarchical integrated control system was built [5]. Before implementing a strategy, it is necessary to perform traffic simulation first and evaluate its performance in traffic environment [6,7]. Car-following models are important components of microscopic traffic simulation, which can present the behaviour of a vehicle when it is following the vehicle in front $[8,9]$.
An appropriate space gap between vehicles in the carfollowing state is difficult to determine. Leaving sufficient space with the vehicle in front can ensure safety, but this gap increases the probability of cut-ins by other vehicles. Dou et al. [10] proposed an overall objective function to find the optimal solution. Turning lanes at intersections are high accident-prone and congestion-prone sections. By understanding the car-following behaviour of turning vehicles, improved design and measures are put forward for adoption. Therefore, traffic efficiency and safety could be effectively improved [11-13]. Considering vehicle spacing, speed of the lead vehicle and driver's sensitivity, an improved car-following model was established based on the Next Generation Simulation (NGSIM) dataset. This model was to improve the accuracy of speed prediction, and it would be helpful for the development of driverless technology [14-18]. 
Car-following model has been studied from multiple perspectives for nearly seven decades. It can be classified from two perspectives: traffic engineering aspect and statistical physics aspect. Car-following models from the traffic engineering aspect include Stimulus-Response models, Safety Distance models, Psycho-Physical models, and Artificial Intelligence models. The statistical physics aspect includes Optimal Velocity (OV) models, Intelligent Driver models, and Cellular Automata models. Among them, the OV model was proposed by Bando et al. [19] to describe the stop-and-go phenomenon in traffic flow. Through optimizing the velocity of the following car, the stability condition of the traffic flow was obtained. The optimized velocity function was constructed in the OV model based on the distance to the preceding vehicle.

The core idea of the OV model is to optimize the optimal velocity of the following car according to headway spacing. It is not sufficient to only consider the effect of the single factor on the car-following behaviour. Hence, traffic scholars have added some variables to the OV model such as headspace, velocity difference, and the acceleration characteristics of the leading vehicle [20-22]. The results showed that the improved model can better describe the change of the acceleration of the leading car.

A new car-following model was established to address the effect of the optimal velocity changes with memory. Both linear stability and nonlinear analyses were performed. And it was found that the stability of the traffic flow could be enhanced by considering the influence of memory on the optimal velocity of car-following behaviour [23]. Based on the OV model, the effect of heterogeneous maximum speeds to the stability of traffic flow was analyzed. The results showed that strengthening the anticipation driving behaviour could enhance the stability of heterogeneous traffic system. Moreover, different stabilities at high or low traffic densities are caused by increasing the value of maximum speed $V_{\max }$ [24].

It was also found that the following vehicle is influenced by the leading vehicle and itself, which can be expressed by the space headway [25]. On the basis of the OV model, an improved car-following model was established and its stability was analyzed. Yang proposed an improved optimal velocity function, considering not only the effect of the space headway but also the influence of the relative velocity between the leading and following vehicles [26]. The improved model can enlarge the stable area of the traffic flow. In the OV model, only using the space headway and safety distance to optimize the car-following velocity might lead to large deviation [27]. An improved optimized speed model was proposed by adding the velocity of the leading vehicle into the OV model. Yang used the average speed of the traffic flow to replace the maximum speed in the optimized velocity function of the OV model, and the results showed that the modified model can achieve a higher accuracy in describing the relationship among traffic parameters [28].

In recent years, driver information navigation system, intelligent transportation system, and intelligent cruise control system have been developed rapidly. Car-following model is playing a more important role in developing advanced driver-assistance systems by accurately modeling driver's car-following behaviour from a microscopic perspective $[3,29,30]$. With the rapid development of information and communication technology, intelligent transportation systems (ITS), information exchange between vehicles, especially the development of connected vehicle and $5 \mathrm{G}$ network, the traditional traffic mode is being subverted and new traffic situations are being created [31-33]. This puts forward a higher requirement for refining the car-following model. Hao et al. [34] applied the datadriven models and the rough set theory to identify hidden information of car-following behaviour. A car-following behavioural decision algorithm was proposed to determine the follower's velocity, which overcame the obstacle that the conventional method only considers ideal traffic conditions.

However, most of the existing models are suitable for the straight traffic, which are difficult to accurately describe the car-following behaviour of turning vehicles at intersections. The car-following behaviour of turning vehicles is significantly different with that of through vehicles, due to the influence of turning radius, sideway force coefficient, and other related factors. Hence, it is necessary to improve the classical model according to the differentiated influencing factors. In this paper, the turning radius of intersection and sideway lateral force coefficient will be introduced into the OV model, and a Turning Optimal Velocity (TOV) carfollowing model at intersections will be proposed. The linear stability analysis and numerical simulation experiments will be carried out to verify the validity of the proposed model.

\section{Developing the Car-Following Model for Turning Vehicles}

2.1. Variable Description and Characteristic Analysis. Taking left-turn vehicles at intersections as an example, the basic scenario of car-following behaviour is shown in Figure 1. The turning process can be divided into three stages: initial acceleration, the formation of the car-following queue, and queue dissipation. The turning vehicles are denoted as $1,2,3, \ldots, n-1, n, n+1$, according to the position sequence in the lane. For example, vehicle $(n)$ follows vehicle $(n-1)$ and vehicle $(n+1)$ follows vehicle $(n)$ in the platoon. The position of vehicle $n$ is denoted as $x_{n}$, and its speed is denoted as $v_{n}$.

In the initial acceleration stage, the following cars, which are influenced by the vehicle operation rules, are more likely to travel at the similar speed of the leading car. To minimize the speed difference among vehicles, drivers might pay less attention to their current speeds. During car-following process, they might pay more attention on the headway space from the vehicle ahead to ensure driving safety. In the dissipation process, the headway increases gradually, and the velocities of following cars begin to show some discrete characteristics.

2.2. Optimal Velocity Model. The OV model, which uses acceleration to describe the car-following behaviour, is easy to understand and can accurately present the running state 


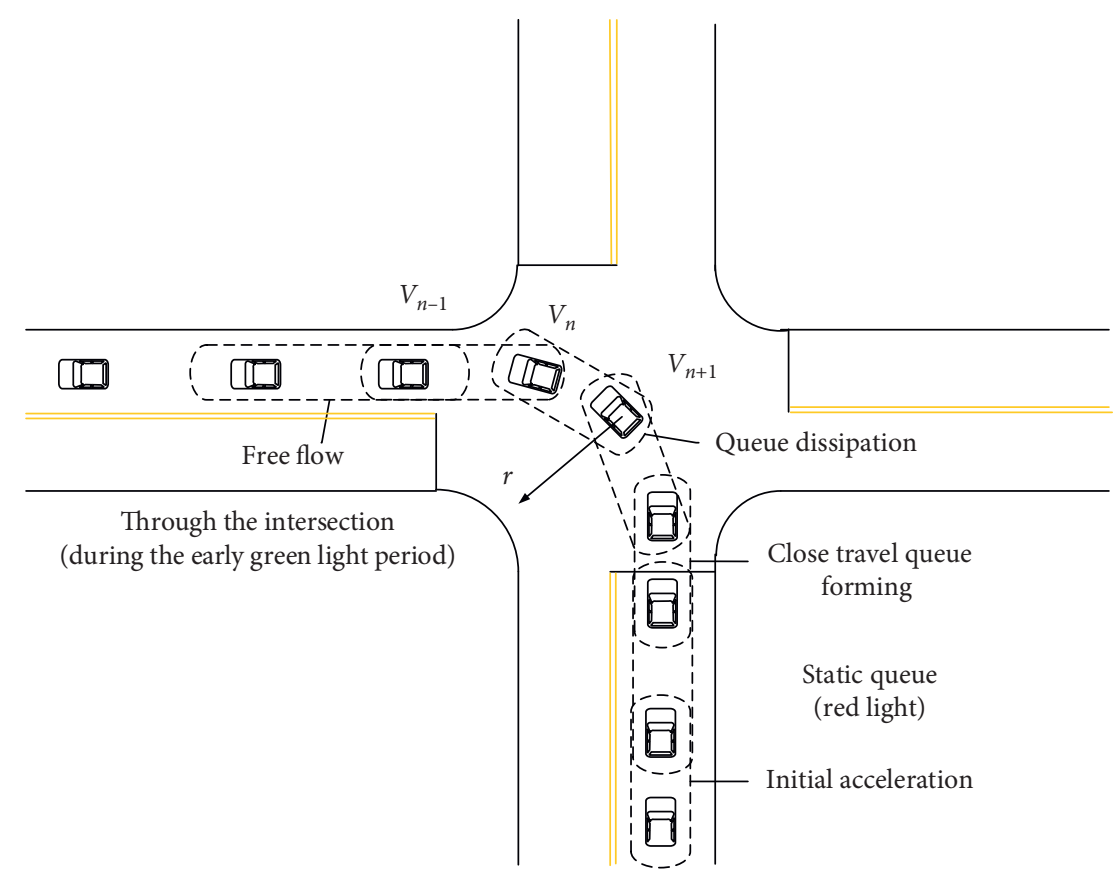

FIgURE 1: The basic mode of turning car-following behaviour at an intersection.

of vehicles in the through lanes. Hence, it conforms pretty well to the traffic flow in reality and becomes one of the most important models to analyze the car-following behaviour. The OV model can be expressed by the changes in acceleration, which is given as follows:

$$
a_{n}(t)=k\left[V\left(\Delta x_{n}(t)\right)-v_{n}(t)\right],
$$

where $a_{n}(t)$ is the acceleration of the following car at time $t$, $\Delta x_{n}(t)$ is the headway of the following car at time $t$, and $v_{n}(t)$ is the speed of the following car at time $t$. The optimal velocity function is represented by $V\left(\Delta x_{n}(t)\right)=V_{\max }\left[\tan h\left(\Delta x-h_{c}\right)+\tan h\left(h_{c}\right)\right] \cdot h_{c}$ is the safe distance between the front car and the following car.

In 1995, Bando first used the optimal velocity function of space headway to determine the dynamic change process of the optimal velocity. And the OV model was established, which can be expressed as follows:

$$
\frac{\mathrm{d}^{2} x_{n}(t)}{\mathrm{d} t^{2}}=k\left[V\left(\Delta x_{n}(t)\right)-v_{n}(t)\right]
$$

where $k$ is a sensitive coefficient; $x_{n}(t)$ represents the position of the $n$th vehicle at time $t ; \Delta x_{n}(t)$ represents the distance between the $n$th vehicle and the vehicle ahead; $v_{n}(t)$ represents the speed of the $n$th vehicle at time $t$; and $V($. represents the optimized velocity function of the vehicle as follows:

$$
V\left(\Delta x_{n}(t)\right)=\frac{V_{\max }}{2}\left[\tan h\left(\Delta x_{n}(t)-h_{c}\right)+\tan h\left(h_{c}\right)\right],
$$

where $v_{\max }$ is the maximum vehicle speed and $h_{c}$ is the safe distance between the vehicle and the vehicle in front. Generally, when $\Delta x_{n} \longrightarrow 0, V\left(\Delta x_{n}\right) \longrightarrow 0$ for avoiding a collision; when $\Delta x \longrightarrow \infty, V\left(\Delta x_{n}\right) \longrightarrow V_{\max }$ means that vehicles can travel at free-flow speed, and there is no interaction between vehicles.

2.3. Turning Optimal Velocity Model for Turning Vehicles at Intersections. The OV model was established mainly for through lanes, which did not consider turning radius, road friction coefficient, and other turning-related factors. Therefore, it could not accurately describe the car-following behaviour of turning at intersections. And thus, Turning Optimal Velocity (TOV) car-following model was established for turning vehicles at an intersection:

$$
\frac{\mathrm{d}^{2} x_{n}(t)}{\mathrm{d} t^{2}}=k\left[V\left(\Delta x_{n}(t), r\right)-v_{n}(t)\right]
$$

where the speed function optimized for turning $V\left(\Delta x_{n}(t), r\right)$ is a function that is related to $\Delta x_{n}(t)$ and $r$ at the same time. Therefore, the optimal velocity function can be expressed by the variable of turning radius. In order to prevent vehicle sliding or overturning in the turning lane, $V_{\max }$ needs to satisfy equation (5) according to the minimum radius (in the design standard):

$$
\frac{V_{\max }^{2}}{127 r}=\mu \pm i,
$$

where $\mu$ is the sideway force coefficient. According to the highway engineering technical standard, the value range of $\mu$ is $0.035 \sim 0.15$. And, $i$ is the super elevation.

As to left turn lanes at intersections, the super elevation can be ignored; thus, $V_{\max }$ can be expressed as follows:

$$
V_{\max }=\sqrt{127 r \mu} \text {. }
$$

Substituting equation (6) in equation (3), we obtain 


$$
V\left(\Delta x_{n}(t)\right)=\frac{\sqrt{127 r \mu}}{2}\left[\tan h\left(\Delta x_{n}(t)-h_{c}\right)+\tan h\left(h_{c}\right)\right]
$$

\section{Description of the Car-Following Behaviour of Turning at Intersections}

In order to accurately examine the car-following characteristics of turning vehicles at an intersection, an actual scene was selected for analysis. On April 18, 2018, the traffic data of left turning vehicles were collected using a digital video camera, at the intersection of Xincheng Avenue and Boxue Road, Changchun City, including a total of 67 cycles and 804 vehicles. An individual photo was extracted every five frames (at $0.2 \mathrm{~s}$ interval) of a video clip. Left-turn vehicles were numbered sequentially $(1,2, \ldots, n, n+1, \ldots)$. Using camera calibration and trajectory extraction software, the parameters of car-following space headway, vehicle acceleration and speed, and the geometric design parameters were extracted to calculate the average values of parameters for each cycle.

Professor Ibuyama of the Kyoto University of Japan found that, under complex road conditions, significant changes can be made in driver's body function because driver's nerve center and sympathetic nerve are being stimulated by overcomplicated traffic information. The results showed that the pulse would increase, the blood flow rate would accelerate, and the blood pressure would rise in challenging driving conditions.

The influence of turning lane design factors on vehicle operation at intersections is mainly reflected in vehicle velocity and acceleration. In order to make a safe turn, drivers must drive carefully so as not to take emergency avoidance or braking measures in case of sudden accidents. For safety reasons, critical values of maximum speed at different turning radii were suggested, as shown in Figure 2. Because the square of speed is proportional to the turning radius and centrifugal acceleration, speed increases rapidly with the increase of turning radius and centrifugal acceleration, resulting in side slipping or overturning.

In Figure 2(a), it can be seen that, at an intersection, with the increase of turning radius, the recommended maximum velocity of motor vehicles increases gradually. It is also noted that when turning radius is greater than $20 \mathrm{~m}$, the maximum velocity of the following vehicle $(n)$ increases slowly. This suggests that when turning radius is greater than $20 \mathrm{~m}$ at intersections, it might be not be an effective way to increase the turning radii to accommodate higher speeds. From Figure 2(b), it shows that when the turning radius is 40 , the peak value of speed distribution frequency appears at $25 \mathrm{~km} / \mathrm{h}$ in the observation samples, and the maximum value is $35 \mathrm{~km} / \mathrm{h}$, which is consistent with Figure 2(a).

\section{Stability Analysis and Verification}

4.1. Stability Analysis. In order to verify the effectiveness of the model, PreScan simulation software was used in this study. By using the GUI and the traffic environment model (including grassland and trees), infrastructure model (including some highway sections, signs, and buildings), traffic actors' model (including various vehicle models and pedestrian models), and sensor models (laser/lidar, camera, millimeter wave radar, infrared, etc.), the turning traffic scene at intersection was built. Millimeter wave radar, laser radar, and vehicle communication sensor were installed on the vehicle to obtain the surrounding driving environment parameters. Building the control system model correctly is the core to demonstrate the normal driving of vehicles properly. The simulation model of the control system was established in Simulink window, and then the model and control algorithm were transformed into Simulink logic diagram. According to the designed Simulink logic diagram and software co-simulation, the driving process and effect diagram of the vehicle can be observed in the 3D viewer interface of PreScan, as shown in Figure 3.

According to the car-following characteristics, the linear stability of the car-following queue is directly related to traffic efficiency and safety. It is necessary to determine whether the following vehicles in a platoon are stable or not, when driver's operating characteristics or the geometric design elements of intersection have changed. On the one hand, the driving states of relative vehicles are needed to be determined. For example, if the fluctuation of the distance between two vehicles is large, the car-following fleet will be unstable, and it will be stable otherwise (called local stability). On the other hand, the speed change of the leading vehicle affects the vehicles behind, which has an influence on the stability of the traffic flow. If the velocity fluctuation becomes larger, the car-following queue will be unstable. Otherwise, the car-following fleet will be stable (called asymptotic stability).

Assuming that the initial state of the current traffic flow is steady, all the vehicles move at the same distance $b$ and the optimal speed $V(b)$, which is can be expressed as follows:

$$
x_{n}^{(0)}(t)=b n+c t,
$$

where $b$ is the space headway of steady flow, $b=L / N$, and $V(b)$ is optimization velocity, $c=V(b, r)$.

Suppose that $y_{n}(t)$ is the initial disturbance of departure from the initial state $x_{n}^{(0)}(t)$, the space headway is calculated as follows:

$$
X_{n}(t)=x_{n}^{(0)}(t)+y_{n}(t), \quad\left|y_{n}\right| \ll 1 .
$$

Substitute equation (9) into the TOV model (equation (4)), and the linearized equation (10) is given as follows:

$$
\frac{\mathrm{d}^{2} y_{n}(t)}{\mathrm{d} t^{2}}=k\left[V^{\prime}(b, r) \Delta y_{n}(t)-\frac{\mathrm{d} y_{n}(t)}{\mathrm{d} t}\right],
$$

where $\Delta y_{n}(t)=y_{n+1}(t)-y_{n}(t), V^{\prime}(b, r)$ is the derivative of the optimal velocity function $V\left(\Delta x_{n}(t), r\right)$ at $\Delta x_{n}(t)=b$, which is shown as follows:

$$
V^{\prime}(b, r)=\left.\frac{\mathrm{d} V\left(\Delta x_{n}(t), r\right)}{\mathrm{d}\left(\Delta x_{n}(t), r\right)}\right|_{\Delta x(t)=b} .
$$

Following the expansion of $y_{n}(t)=\exp \left(i a_{k_{n}}+z t\right)$, equation (12) about $z$ is obtained as follows: 


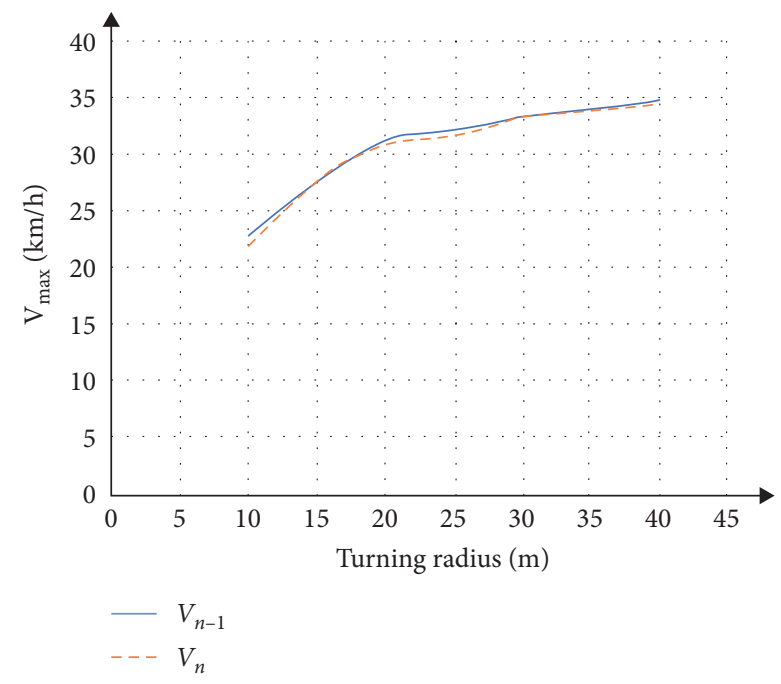

(a)

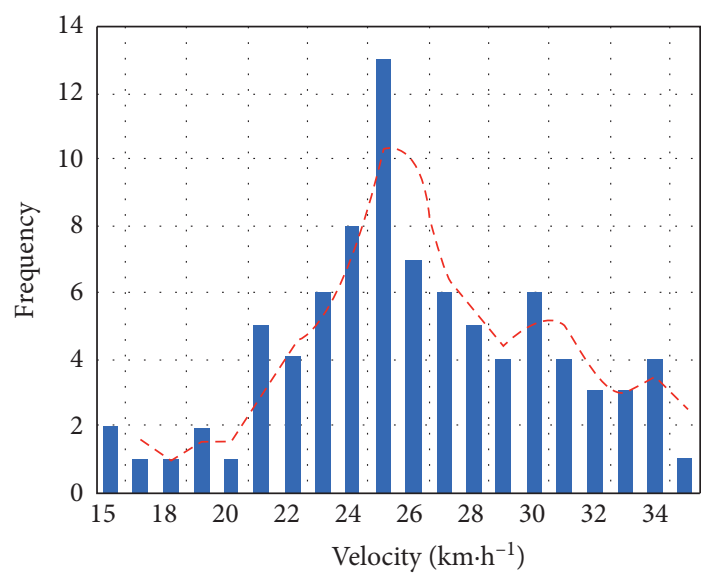

(b)

FIgURE 2: The relationship between velocity and radius: (a) variation of maximum velocity under different turning radii and (b) the frequency distribution of velocity, when radius is $40 \mathrm{~m}$.

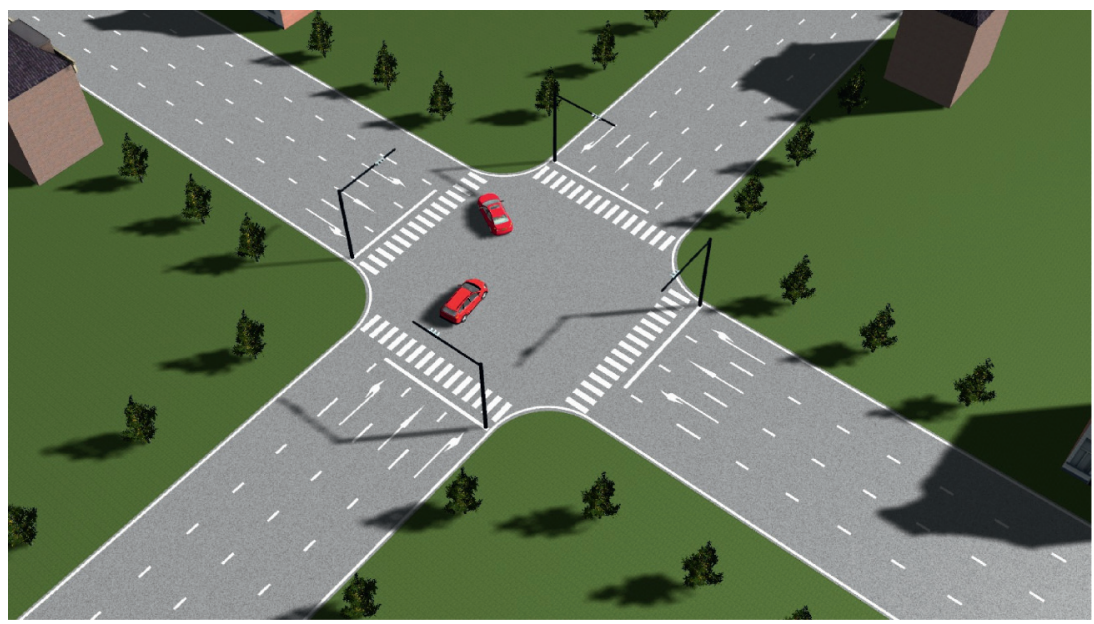

Figure 3: Simulation of the car-following process of turning vehicles at an intersection.

$$
z^{2}+k Z-k V^{\prime}(b, r)\left(e^{i \alpha_{k}}-1\right)=0
$$

Based on the long wave expansion, the stability condition of the model is derived by substituting into equation (12), and equation (13) is obtained:

$$
\begin{aligned}
& \lambda^{2}-\omega^{2}+k \lambda-k V^{\prime}(b, r)\left(\cos \alpha_{k}-1\right) \\
& \quad+i\left[2 \lambda \omega+k \omega-k V^{\prime}(b, r) \sin \alpha_{k}\right]=0 .
\end{aligned}
$$

Suppose $\lambda=0$, and equation (14) is obtained: $-\omega^{2}-k V^{\prime}(b, r)\left(\cos \alpha_{k}-1\right)+i\left[k \omega-k V^{\prime}(b, r) \sin \alpha_{k}\right]=0$.

The real and imaginary parts of equation (14) are set to 0 , and equations (15) and (16) can be obtained:

$$
\begin{aligned}
-\omega^{2}-k V^{\prime}(b, r)\left(\cos \alpha_{k}-1\right) & =0, \\
k \omega-k V^{\prime}(b, r) \sin \alpha_{k} & =0,
\end{aligned}
$$

where $\omega$ can be expressed as shown in the following equation:

$$
\omega=V^{\prime}(b, r) \sin \alpha_{k}
$$

Substituting equation (17) into equation (15), we obtain

$$
V^{\prime}(b, r) \sin ^{2} \alpha_{k}+k\left(\cos \alpha_{k}-1\right)=0,
$$

where $V^{\prime}(b, r)$ can be shown as

$$
V^{\prime}(b, r)=\frac{k\left(1-\cos \alpha_{k}\right)}{\sin ^{2} \alpha_{k}}
$$


At the same time, let $\alpha_{k} \longrightarrow 0$, the linear equation of $L \prime$ Hospital rule is applied to the critical stability curve:

$$
V^{\prime}(b, r)=\frac{k}{2}
$$
by

The stability conditions of the TOV model are expressed

$$
V^{\prime}(b, r)<\frac{k}{2}
$$

When the stability conditions (equation (21)) are not satisfied, a small disturbance imposed on a uniformly distributed traffic stream will lead to traffic jams due to linear instability.

The derivation of $V(b, r)$ can be obtained by

$$
V^{\prime}(b, r)=\frac{\sqrt{127 r \mu}}{2\left[\cos h\left(b-h_{c}\right)\right]^{2}} .
$$

Substituting equation (22) into equation (21), we obtain

$$
\frac{\sqrt{127 r \mu}}{2\left[\cos h\left(b-h_{c}\right)\right]^{2}}<\frac{k}{2} .
$$

According to the linear stability condition (equation (23)), it is noticed that the space headway between the following and leading cars of turning is an independent variable, and the sensitivity coefficient (unit: $\mathrm{s}^{-1}$ ) of driver's response is a dependent variable, which reflects the change of driver response caused by the change of independent variable $(r)$ in the car-following model. Let the parameter $h_{c}=4 \mathrm{~m}$, the turning radii of intersection are $r=20 \mathrm{~m}$, $r=30 \mathrm{~m}$, and $r=50 \mathrm{~m}$, and the sideway force coefficient at the intersection is 0.04 . Under various weather conditions, speed limit is a necessary measure to ensure traffic safety. Therefore, the maximum speed $V_{\max }$ is directly related to the road friction coefficient. The medium linear stability conditions of the TOV car-following model is shown in Figure 4. The upper part of the linear stability curve presents the stable area of traffic stream, and the lower part shows the unstable one. When $r=30 \mathrm{~m}$, driver's critical response sensitivity coefficient $k_{0} \approx 4.8$. If $k>k_{0}$, the traffic stream is stable, disturbances will disappear over time. And the traffic flow is unstable, otherwise.

In Figure 3, driver's sensitivity coefficient decreases gradually and the stable area of traffic flow increases gradually with the decrease of turning radius $(r)$. Compared with the stability condition of the OV model, the traffic flow becomes more stable when the turning radius is taken into account, and it is more suitable to describe the car-following behaviour under turning conditions.

When the turning radius of intersection is fixed, the sensitivity coefficient increases rapidly and then decreases rapidly with the increase of distance between vehicles. This is because when the distance between the front and rear vehicles is lower than the safety distance. It means that the front and rear vehicles are very close, and the two vehicles are running at low speed or queuing state. When the vehicle stops completely, although the distance between the front

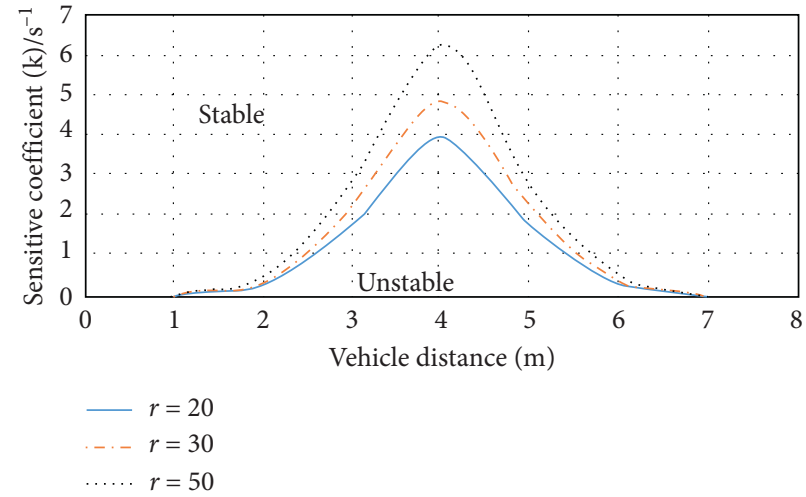

FIgURE 4: Medium linear stability conditions of the TOV carfollowing model.

and rear vehicles is very small, the driver's response sensitivity is very low. When the distance between the front and rear vehicles increases gradually and approaches the safe distance gradually, the driver's sensitivity coefficient gradually increases. Drivers always pay attention to the dynamical changes of the front vehicle and make corresponding response, accelerating with the acceleration of the front vehicle or decelerating with the deceleration of the front vehicle. When the distance between two vehicles exceeds the safety distance, the driver response sensitivity coefficient is also at a relatively high value. However, with the increase of distance, the influence of the running state of the front vehicle on the running state of the rear vehicle gradually decreases, and the sensitivity coefficient gradually decreases until it tends to a small value.

4.2. Numerical Simulation. In order to further illustrate the stability of the traffic flow in the TOV car-following model and verify the correctness of the theoretical analysis, the corresponding numerical simulation experiments were carried out. Under the critical conditions, the length of turn lane $L=100 \mathrm{~m}$, the total number of vehicles $N=4$, the average distance $b=10 \mathrm{~m}$, and the initial velocity $v_{0}=8.88 \mathrm{~m} / \mathrm{s}$, the initial position and disturbance of the vehicle are shown as follows:

$$
\begin{aligned}
& x_{1}(0)=1 m, \\
& x_{n}(0)=h(n-1), \quad n=2,3, \ldots, N .
\end{aligned}
$$

Given driver response sensitivity coefficient $k_{0} \approx 4.8$, the turning radius $r=30 \mathrm{~m}$ were selected for simulation experiments. The simulation results are shown in Figures 5(a) and $5(\mathrm{~b})$.

As shown in Figure 5, it can be seen that when the initial disturbance is $x_{1}(0)=1 \mathrm{~m}$, the velocity of the following vehicle fluctuates noticeably, which transmits backward over time. When the range of velocity fluctuation is controlled within $3 \%$, the traffic stream can be considered to be stable. The evolution of traffic stream's velocity with time in the OV model is shown in Figure 5(a). It was seen that the fluctuation of velocity decreases over time, but it does not change much, which 


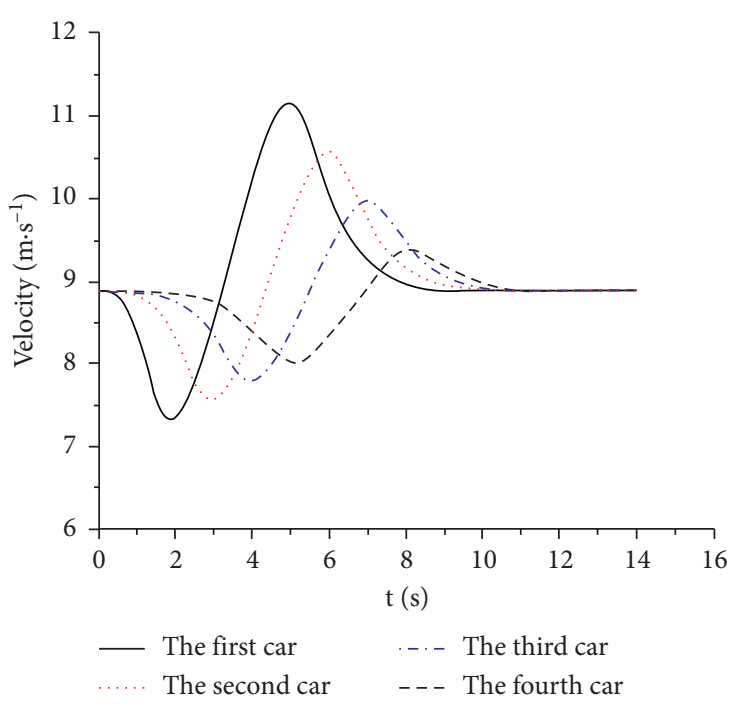

(a)

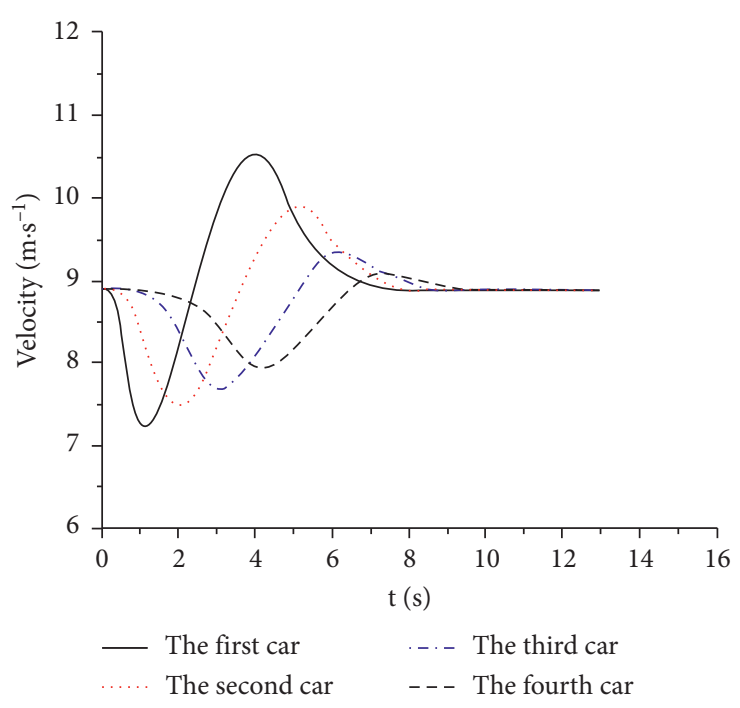

(b)

Figure 5: The fluctuation of velocities in different car-following models: (a) the fluctuation of velocity in OV model and (b) the fluctuation of velocity in TOV model.

means that the disturbance is absorbed slowly. When $t=9.7 \mathrm{~s}$, the maximum velocity is $v=9.15 \mathrm{~s}$ with an upward fluctuation of $3 \%$ and the minimum velocity is $v=8.65 \mathrm{~m} / \mathrm{s}$ with a downward fluctuation of $2.57 \%$. At this time, the system basically reaches a stable state.

Figure 5(b) shows the evolution of vehicle speed with time, under the influence of the initial disturbance in the TOV model. The velocity fluctuation curve shows that when the initial disturbance is $x_{1}(0)=1 \mathrm{~m}$, the speeds of the following vehicles also fluctuate in the same trend, which transmit backward over time. Under the same initial disturbance, the initial deceleration processes of the vehicles are similar. In the subsequent acceleration and deceleration processes, the speed fluctuation amplitude of the TOV model is smaller than that of the OV model, and it can return to the stable state faster.

Obviously, compared to Figure 5(a), the speed of absorbing disturbances is faster and the range of speed fluctuation is smaller. When $t=8.0 \mathrm{~s}$, the maximum velocity is $v=9.13 \mathrm{~m} / \mathrm{s}$ with an upward fluctuation of $2.86 \%$, and the minimum velocity is $v=8.69 \mathrm{~m} / \mathrm{s}$ with a downward fluctuation of $2.14 \%$. At the moment, the system reaches a steady state. Therefore, compared to the $\mathrm{OV}$ model, the time required for the system to reach a stable state is shorter, and the speed of absorbing disturbances is faster. The essential reason for this phenomenon is that drivers are more careful and precise in controlling vehicle speed than in straight road section under the influence of turning radius at intersection. In the process of turning at the intersection, when the front vehicle decelerates or accelerates, the driver of the rear vehicle quickly makes judgment and corresponding operation to ensure the stability of the car-following process. Therefore, the use of turning radius as a key variable in the car-following process can more properly reflect the driving behaviour characteristics in the scene.
The simulation results show that the turning radius has a strong influence on the stability of turning traffic stream at intersections. Under the influence of disturbance, the TOV model with consideration of turning radius reaches a more stable state than the OV model. It indicates that the model can describe the car-following behaviour more accurately with consideration of turning radius.

\section{Conclusion}

This study analyzed the characteristics of car-following behaviour in turning process at intersections and proposed a Turning Optimal Velocity (TOV) car-following model with consideration of turning radius. The main findings are summarized as follows:

(1) With consideration of the turning radius, the sideway force coefficient, and other turning-related factors at intersections, the TOV car-following model can more accurately describe the car-following behaviour of vehicles in a turning process.

(2) Linear stability analysis of the TOV car-following model shows that the stable area of traffic stream increases with the decrease of the turning radius of intersection. With the increase of turning radius, the following vehicle is more sensitive to the stimulation of the car in front of it. The turning radius of intersection has a significant effect on the stability of the traffic flow. Numerical simulation experiments further verify the influence of turning radius on the stability of turning traffic flow at intersections. This paper determined the performance and description of car-following behaviour under the turning condition at intersections. It effectively shows the influence of turning radius and other key factors on car-following behaviour. These findings can be used 
in the development of traffic simulation software and for improving traffic safety at intersections. In future work, we will be interested in analysing the effect on right-turn traffic flow at intersections. We will also be interested in improving the TOV car-following model by considering more factors, such as weather condition and super-high transverse gradient of highway.

\section{Data Availability}

The data used to support the findings of this study are included within the article.

\section{Conflicts of Interest}

The authors declare that there are no conflicts of interest regarding the publication of this paper.

\section{Acknowledgments}

This work was supported by the National Natural Science Foundation (Grant nos. 71901134 and 51942806), National Science Fund for Distinguished Young Scholars (Grant no. 51925081), and Jiangsu Postdoctoral Research Funding Scheme (Grant no. 2018K118C).

\section{References}

[1] C. Sun, Y. Luo, and J. Li, "Urban traffic infrastructure investment and air pollution: evidence from the 83 cities in China," Journal of Cleaner Production, vol. 172, pp. 488-496, 2018.

[2] J. Beaudoin, Y. H. Farzin, and C.-Y. C. Lin Lawell, "Public transit investment and sustainable transportation: a review of studies of transit's impact on traffic congestion and air quality," Research in Transportation Economics, vol. 52, pp. 15-22, 2015.

[3] Z. Zhou, Y. Zhou, Z. Pu, and Y. Xu, "Simulation of pedestrian behavior during the flashing green signal using a modified social force model," Transportmetrica A: Transport Science, vol. 15, no. 2, pp. 1019-1040, 2019.

[4] D. Ma, X. Luo, S. Jin, D. Wang, W. Guo, and F. Wang, "Lanebased saturation degree estimation for signalized intersections using travel time data," IEEE Intelligent Transportation Systems Magazine, vol. 9, no. 3, pp. 136-148, 2017.

[5] R.-H. Zhang, Z.-C. He, H.-W. Wang, F. You, and K.-N. Li, "Study on self-tuning tyre friction control for developing main-servo loop integrated chassis control system," IEEE Access, vol. 5, pp. 6649-6660, 2017.

[6] D. Xu, C. Wei, P. Peng, Q. Xuan, and H. Guo, "GE-GAN: a novel deep learning framework for road traffic state estimation," Transportmetrica Research Part C: Emerging Technologies, vol. 117, Article ID 102635, 2020.

[7] M. Zhou, Y. Yu, and X. Qu, "Development of an efficient driving strategy for connected and automated vehicles at signalized intersections: a reinforcement learning approach," IEEE Transactions on Intelligent Transportation Systems, vol. 21, no. 1, pp. 433-443, 2020.

[8] J. Zhao and P. Li, "An extended car-following model with consideration of vehicle to vehicle communication of two conflicting streams," Physica A: Statistical Mechanics and Its Applications, vol. 473, pp. 178-187, 2017.
[9] U. Durrani, C. Lee, and H. Maoh, "Calibrating the Wiedemann's vehicle-following model using mixed vehicle-pair interactions," Transportation Research Part C: Emerging Technologies, vol. 67, pp. 227-242, 2016.

[10] Y. Dou, D. Ni, Z. Wang, J. Wang, and F. Yan, "Strategic carfollowing gap model considering the effect of cut-ins from adjacent lanes," IET Intelligent Transport Systems, vol. 10, no. 10 , pp. 658-665, 2016.

[11] C.-G. J. Gerardo, A.-L. Jesús, and O.-M. Ricardo, "Modeling the turning speed and car following behaviors of autonomous vehicles in a virtual world," Ingeniería, Investigación $Y$ Tecnología, vol. 16, no. 3, pp. 391-405, 2015.

[12] C. Xu, Y. Yang, S. Jin, Z. Qu, and L. Hou, "Potential risk and its influencing factors for separated bicycle paths," Accident Analysis \& Prevention, vol. 87, pp. 59-67, 2016.

[13] R. Guo, C. Xin, P.-S. Lin, and A. Kourtellis, "Mixed effects logistic model to address demographics and neighborhood environment on pedestrian injury severity," Transportation Research Record: Journal of the Transportation Research Board, vol. 2659, no. 1, pp. 174-181, 2017.

[14] S. Jiao, S. Zhang, Z. Li, B. Zhou, and D. Zhao, "An improved car-following speed model considering speed of the lead vehicle, vehicle spacing, and driver's sensitivity to them," Journal of Advanced Transportation, vol. 2020, Article ID 279420, 13 pages, 2020.

[15] Y. Wei, C. Avc1, J. Liu et al., "Dynamic programming-based multi-vehicle longitudinal trajectory optimization with simplified car following models," Transportation Research Part B: Methodological, vol. 106, pp. 102-129, 2017.

[16] X. Qu, Y. Yu, M. Zhou, C.-T. Lin, and X. Wang, "Jointly dampening traffic oscillations and improving energy consumption with electric, connected and automated vehicles: a reinforcement learning based approach," Applied Energy, vol. 257, Article ID 114030, 2020.

[17] Z. Ning, Y. Feng, M. Collotta et al., "Deep learning in edge of vehicles: exploring trirelationship for data transmission," IEEE Transactions on Industrial Informatics, vol. 15, no. 10, pp. 5737-5746, 2019.

[18] X. Kong, F. Xia, J. Li, M. Hou, M. Li, and Y. Xiang, “A shared bus profiling scheme for smart cities based on heterogeneous mobile crowdsourced data," IEEE Transactions on Industrial Informatics, vol. 16, no. 2, pp. 1436-1444, 2020.

[19] M. Bando, K. Hasebe, A. Nakayama, A. Shibata, and Y. Sugiyama, "Dynamical model of traffic congestion and numerical simulation," Physical Review E, vol. 51, no. 2, pp. 1035-1042, 1995.

[20] A. Reuschel, "Vehicle movements in the column uniformly accelerated or delayed," Oesterrich Ingr Arch, vol. 4, pp. 193-215, 1950.

[21] Z. Jin, Z. Li, R. Cheng, and H. Ge, "Nonlinear analysis for an improved car-following model account for the optimal velocity changes with memory," Physica A: Statistical Mechanics and Its Applications, vol. 507, pp. 278-288, 2018.

[22] Z. Ge, "Numerical simulation of car-following model considering preceding vehicle's acceleration," Computer Engineering and Applications, vol. 51, no. 11, pp. 47-49+66, 2015.

[23] G. Peng, W. Lu, H. He, and Z. Gu, "Nonlinear analysis of a new car-following model accounting for the optimal velocity changes with memory," Communications in Nonlinear Science and Numerical Simulation, vol. 40, pp. 197-205, 2016.

[24] F. Sun, J. Wang, R. Cheng, and H. Ge, "An extended heterogeneous car-following model accounting for anticipation driving behavior and mixed maximum speeds," Physics Letters A, vol. 382, no. 7, pp. 489-498, 2018. 
[25] H. Ge, Y. Cui, and R. Cheng, "A car-following model with considering control signals from front and rear," Acta Physica Sinica, vol. 63, no. 11, pp. 95-101, 2014.

[26] X. Yang, "Car-following model based on improved optimal velocity and its numerical simulation," Computer Application and Software, vol. 32, no. 6, pp. 117-119, 2015.

[27] L. Lai, Y. Hu, and Z. Shi, "Improved speed model based on headway and speed of front vehicle," Journal of Northwestern Polytechnical University, vol. 32, no. 1, pp. 118-122, 2014.

[28] L. Yang, S. Zhao, and H. Xu, "Car-following model based on the modified optimal velocity function," Journal of Transportation Systems Engineering and Information Technology, vol. 17, no. 2, pp. 41-46+67, 2017.

[29] M. Mai, L. Wang, and G. Prokop, "Advancement of the car following model of Wiedemann on lower velocity ranges for urban traffic simulation," Transportation Research Part F: Traffic Psychology and Behaviour, vol. 61, pp. 30-37, 2019.

[30] D. Ma, X. Song, and P. Li, "Daily traffic flow forecasting through a contextual convolutional recurrent neural network modeling inter-and intra-day traffic patterns," IEEE Transactions on Intelligent Transportation Systems, vol. 99, 10 pages, Article ID 2973279, 2020.

[31] Y. Guo, X. Wang, Q. Xu, F. Liu, Y. Liu, and Y. Xia, "Changepoint analysis of eye movement characteristics for female drivers in anxiety," International Journal of Environmental Research and Public Health, vol. 16, no. 7, p. 1236, 2019.

[32] Z. Ning, R. Y. K. Kwok, K. Zhang et al., "Joint computing and caching in 5 g-envisioned internet of vehicles: a deep reinforcement learning based traffic control system," IEEE Transactions on Intelligent Transportation Systems, 12 pages, Article ID 2970276, 2020.

[33] S. L. Vine, A. Zolfaghari, and J. Polak, "Autonomous cars: the tension between occupant experience and intersection capacity," Transportation Research Part C, vol. 5, pp. 1-14, 2015.

[34] S. Hao, L. Yang, and Y. Shi, "Data-driven car-following model based on rough set theory," IET Intelligent Transport Systems, vol. 12, no. 1, pp. 49-57, 2018. 